\title{
A Toolkit to Assess the Covit-19 Syndrom
}

\author{
A Short Communication
}

\author{
Rafaat Hussein \\ State University of New York - ESF \\ Syracuse, NY 13210, USA \\ email:rmhussei@esf.edu
}

Abstract: Report after report reinforces the prevailing vulnerability of human health to the COVIT-19 that must be tackled worldwide. Using tracking and assessment systems can assist the authority to achieve this goal. This communication is a step for enhancing the effectiveness of the existing tackling approaches. The overall long-term goal for this research effort is to develop a robust monitoring system to rapidly detect the adverse impacts in pandemic(s). The overarching goal includes:

- $\quad$ Vulnerability and risk assessment.

- $\quad$ Management and protection strategies.

The COVIT-19 issue is inherently complex and has no unique methodology so far to address it. However, we believe that the computer knowledge-based technologies provide the adequate tools needed for probing and assessing the level of vulnerability and tracking its impacts (1, $2,3)$. In this communication, we termed the situation as syndrome based on its widespread adverse impacts.

Keywords: Covit-19, epidemic, pandemic, trajectory, vulnerability.

\section{INTRODUCTION}

In its report on April 7, 2020, the US' Centers for Disease Control and Prevention (CDC) (4) indicated that the COVID19 poses a serious public health and the severity of the pandemic can vary from one area to another. In its report, the CDC indicated that the "complete clinical picture with regard to COVID-19 is not fully known." That information agrees with our previous perspective of the complexity of this issue. The COVID-19 is new and its pandemic is the first to be caused by a new coronavirus. Since its appearance, a great deal of research was conducted and the World Health Organization (WHO) has created an overwhelming pertinent database (5). Many other organizations have also provided useful resources, statistics, and information including the Chinese Medical Association Publishing House (6), the European Centre for Disease Prevention and Control (7), to name a few examples. The CDC provides a daily report that includes data reported to the CDC by 55 USA jurisdictions (8).

The available data may suggest that the number of COVIT-19 cases and deaths play a key role in the analysis that is vital to assess the vulnerability of different regions to the virus and mapping its trajectory in order to forecast the future hit areas. The daily news on COVIT-19 suggests inconsistent reports from various parts of the world. This may be due to the large number of pertinent data, the diverse global understanding of the virus and how to stop it, the overwhelming number of parties that deal with this new occurrence, and wide scope of cultural issues. As an attempt to apply a sound assessment and tracking simulation, a computer toolkit was developed and proposed in this article.

\section{DESCRIPTION OF THE TOOLKITTOOLKIT}

As stated previously, the available literature seems to suggest using the number of reported cases and deaths as the key variables to analyze the COVIT-19 in various ways.

The uncontrolled and seemingly unpredictable spreading of COVIT-19 play key roles in increasing the humans' susceptibility to its adverse effects. This dilemma makes the monitoring and management in any country challenging tasks. To harness the existing and new variables, a new index to assess the vulnerability of various states to the COVIT-19 and to track its trajectory. This in-progress simulation expanded the scope of that analysis by considering additional variables such as the areas in which the cases and deaths are recorded. In addition, the impacts of the virus on different regions should be compared to assess the level of severity and potential vulnerability.

Because the COVIT-19 syndrome is pervasive encompassing a large number of unsolved global challenges, we propose a seed concept for a promising simulation for the identification, assessment, and monitoring strategies. It suggests a novel digital toolkit the puts the overall syndrome in perspective that is easy to comprehend, facilitating the analysis of the COVIT19 data, to effectively use it in making sound decisions, and to enable any person to engage in concerted efforts to stop the spreading that will save the life of million people. This proposal may serve as a platform from which pertinent future advances are launched. In general, the toolkit is useful for:

1. Identification of vulnerable regions.

2. Quantification of severity.

3. Prediction of the trajectory. 
Figure 1 shows the user friendly, interactive, graphical, intuitive dashboard of the toolkit. It was developed using the available data on March 27, 2020 and April 4, 2020. A database was developed using the available county-by-county data on COVIT-19 in the eight most vulnerable states in the US: New York, Florida, California, Texas, Louisiana, Washington, Colorado, Georgia, Illinois, New Jersey, and Michigan. The results indicate that the vulnerability index increased for all states except for Michigan State. New York State index increased by $78 \%$ whereas the index for Washington State increased by $60 \%$ in the same time frame. On April 4, the two most vulnerable states were NY and WA, followed by LA then IL and NJ. Other lessons can be extracted from the dashboard.

\section{SUMMARY}

It is unrealistic to find nowadays a human who has not heard about COVIT-19. The available pertinent information indicates its unsafe and economical challenges worldwide. The global spreading of the new corona virus could be contained using strategies designed to curb its vulnerability. It was this recognition that prompted this conceptual proposal in this communication from the emphasis on the row data.

\section{REFERENCES}

[1] Hussein, R., "Knowledge-based \& expert system technologies for built \& natural environments," https://aitechm.wixsite.com/8kbextech, 2020.

[2] Hussein, R., "Artificial Intelligence for the Built and Natural Environments and Climate," Chapter 7, Advances in Artificial Intelligence, edited by Sergey Yurish, the International Frequency Sensor Association, 2019.

[3] Hussein, R., "Knowledge-based Tools for Monitoring and Management of the Engineered Infrastructure Construction Systems," Advances in Computers and Software Engineering, edited by Sergey Yurish, Chapter 5, International Frequency Sensor Association, 2020.

[4] The Centers for Disease Control and Prevention [CDC], "Situation Summary," https://www.cdc.gov/coronavirus/2019ncov/cases-updates/summary.html, April 7, 2020.

[5] The World Health Organization [WHO], "Global research on coronavirus disease [COVID-19]," https://www.who.int/emergencies/diseases/novel-coronavirus2019/global-research-on-novel-coronavirus-2019-ncov, April 7, 2020

[6] The Chinese Medical Association Publishing House, "COVID19 Academic Research Platform," http://eng.med.wanfangdata.com.cn/Chinese_Medical_Associati on_Publishing_House.html, April 7, 2020.

[7] The European Centre for Disease Prevention and Control, "Coronavirus disease," https://www.ecdc.europa.eu/en, April 7, 2020
[8] The Centers for Disease Control and Prevention [CDC), "Cases in U.S.," https://www.cdc.gov/coronavirus/2019-ncov/casesupdates/cases-in-us.html, April 7, 2020

covit19

IK TOOLKIT TO ASSESS THE HUMAN HEALTH VULNEDABILITY IN DANDEMIC

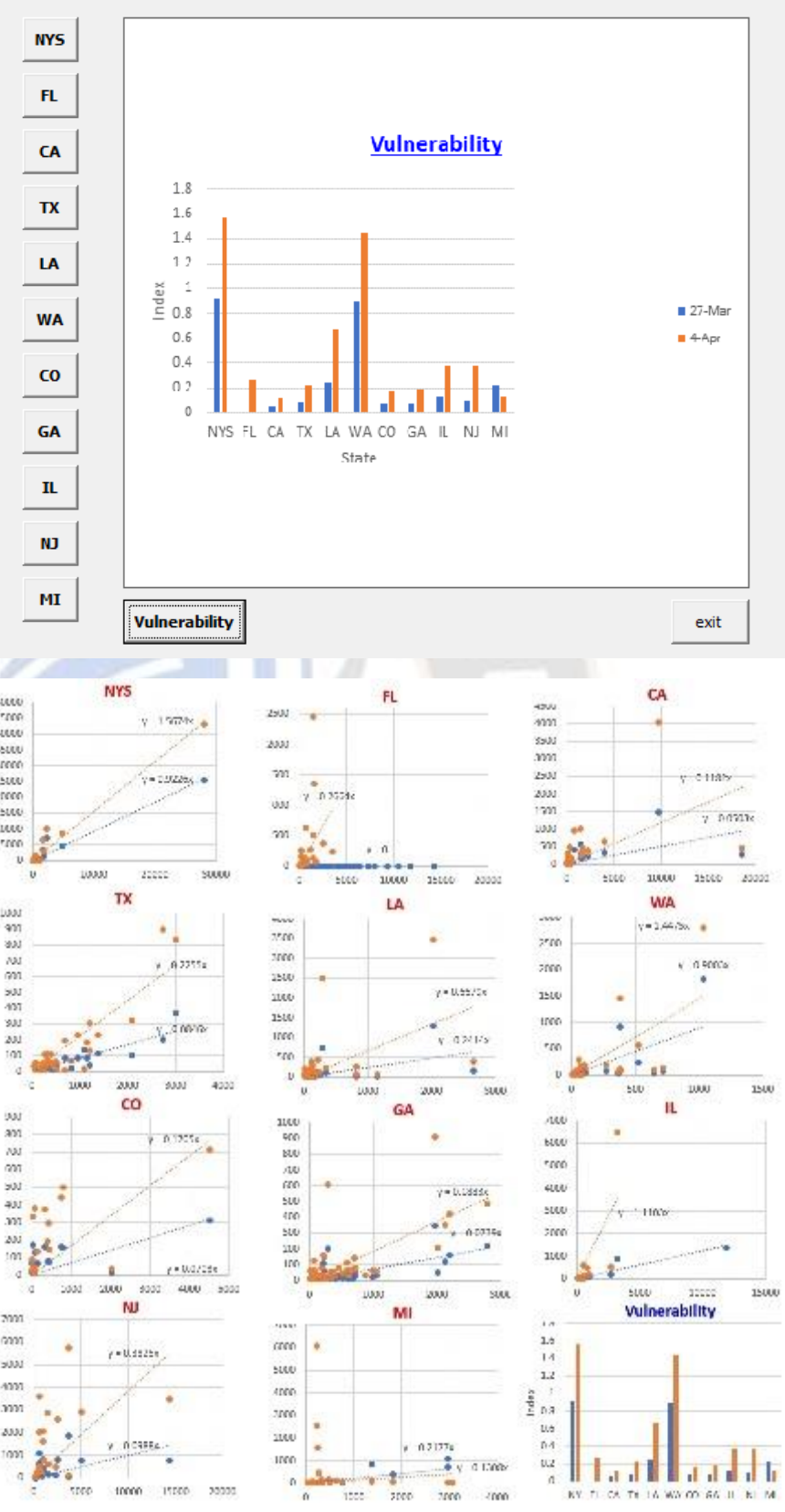

Figure 1: Dashboard of the proposed Toolkit 